Available online on 15.01.2017 at http://jddtonline.info
C 2016, publisher and licensee JDDT, This is an Open Access article which permits unrestricted
noncommercial use, provided the original work is properly cited

Research Article

\title{
LIPOSOMES: DRUG DELIVERY SYSTEM OR POSSIBLE DOPING AGENT?
}

\author{
Nimker Vandana*1,2, Jamal Haseen ${ }^{2}$, Ghosh Prahlad C ${ }^{1}$, Jain Shila ${ }^{2}$, Beotra Alka ${ }^{2}$ \\ ${ }^{1}$ Department of Biochemistry, University of Delhi, South Campus, New Delhi, India \\ ${ }^{2}$ National Dope Testing Laboratory, New Delhi, India
}

\begin{abstract}
The use of liposomes as drug delivery vehicle for treatment of various diseases is well known in medical field but its possible role as a masking agent in sports came into light when Liposom Forte ${ }^{\circledR}$ was found stored together with banned and non-banned drugs during investigations carried out by Italian legal authorities and recent availability of IGF-1 Liposomal Spray on internet. Role of liposomes as masking agent for anabolic steroids in the field of doping has been investigated by Botre et al. The aim of the present work was to study the effect of different parameters like temperature, $\mathrm{pH}$, charge, time, concentration etc. on the interaction of liposomes and doping agents and to identify a possible marker for detection of their abuse in sports. The results showed that out of variety of doping agents, the direct addition of liposomes to urine samples containing anabolic steroids shows strong tendency to interact with the liposomes which results in the reduced concentration of the compound in the sample. However, there was no significant effect of temperature and incubation time on the interaction of liposome and doping agents while other parameters such as charge and concentration of liposome affect the interaction capacity.
\end{abstract}

Keywords: WADA, Liposomes, Doping

Article Info

Received 25 Dec 2016; Review Completed 01 Jan 2017; Accepted 03 Jan 2017, Available online 15 Jan 2017

Cite this article as:

Nimker V, Jamal H, Ghosh PC, Jain S, Beotra A, Liposomes: drug delivery system or possible doping agent?, Journal of Drug Delivery and Therapeutics. 2017; 7(1):25-29, DOI: http://dx.doi.org/10.22270/jddt.v7i1.1369

* Corresponding author:

Vandana Nimker, Research Associate, National Dope Testing Laboratory NDTL), East Gate No. 10, JLN Stadium Complex, Near MTNL Building, Lodhi Road, New Delhi-03, Email Id: vandana.nimker@gmail.com

\section{INTRODUCTION:}

Liposomes are self-assembled lipoidal vesicles used for encapsulation of drugs in drug delivery system. The hydrophobic drugs intercalate in between lipid bilayer while hydrophilic drugs encapsulated in aqueous core of the liposome. This property of liposomes could be misused by athletes to alter the concentration of drugs in urine sample when liposomes were added externally in urine at the time of sample collection. The concept of interaction between liposome and anabolic steroids has been studied and reported by Botre et $\mathrm{al}^{1,2}$. The present work aimed to ascertain the effect of various parameters on in-vitro interaction of liposomes with various threshold and non-threshold substances prohibited in sports; and secondly to identify a possible marker for the detection of liposome abuse.

\section{MATERIALS AND METHOD:}

\section{Reagents and Chemicals}

Reference standards were obtained from SigmaAldrich, USA, and National Measurement Institute (NMI), Australia. Empty liposomes [Coatsomes EL Series: EL-01-A (Anionic ( DPPC: Cholesterol: $\mathrm{DPPG}=30: 40: 30 \mu \mathrm{mol} / \mathrm{vial})$ ), EL-01-C (Cationic (DPPC: Cholesterol: Stearylamine $=52: 40: 8 \mu \mathrm{mol} / \mathrm{vial})$ ) and EL-01-N (Neutral(DPPC: Cholesterol: $\mathrm{DPPG}=54: 40: 6 \mu \mathrm{mol} / \mathrm{vial}))]$ from Nippon Oil \& Fats Co., Ltd (NOF) (Tokyo, Japan), $\beta$ - Glucuronidase from E. coli (Roche Diagnostics, USA) and other reagents, solvents of HPLC or analytical grade were procured. 


\section{Experimental Design}

Each vial of freeze dried liposomes was reconstituted as per manufacturer's specification. Interaction of liposomes was studied with approximately 120 WADA Prohibited Drugs. Out of 120 drugs, 5 threshold compounds (19-Norandrosterone, 19Noretiocholanolone, Morphine, Salbutamol, CarboxyTetrahydrocannabinol) and 6 endogenous steroids (Androsterone, Etiocholanolone, Testosterone, Epitestosterone, $5 \alpha$-androstane- $3 \alpha, 17 \beta$-diol ( $5 \alpha$-diol), $5 \beta$-androstane- $3 \alpha, 17 \beta$-diol $(5 \beta$-diol $))$ were selected to ascertain effect of various parameters i.e. concentration, $\mathrm{pH}$, time, temperature on the interaction efficiency of liposomes. To study the effect of concentration of liposomes on binding efficiency with various compounds, five different volumes (10-100 $\mu \mathrm{l})$ of reconstituted liposomes of different types (anionic, cationic and neutral liposomes) were added to urine samples spiked with drugs at threshold level. To determine the effect of $\mathrm{pH}(\mathrm{pH} \mathrm{5,7}$, and 9), time of interaction ( $24 \& 48 \mathrm{hrs}$ ) and operating temperature (4 ${ }^{0} \mathrm{C}, 37{ }^{0} \mathrm{C} \&$ room temperature (RT $25 \pm 2$ )), compounds were spiked in urine at their threshold level, $40 \mu \mathrm{l}$ of reconstituted liposome of different ionic strength was added in each sample. Entire experiments were repeated for five consecutive days. Post reaction samples were screened using in house extraction procedure for anabolic steroids and analysed on Gas Chromatography-Mass Spectrometry (GC-MSD) ${ }^{3}$.

\section{Monitoring of Cholesterol}

Cholesterol was incorporated in routine method duly validated for quantification. Concentration of cholesterol was measured in 1000 routine samples and samples of liposome interaction experiments.

\section{Data Analysis}

With each experiment one spiked sample was prepared and analyzed without addition of liposomes. Recovery of target compounds was calculated by peak area ratios of each analyte to internal standard.

\section{RESULT AND DISCUSSION:}

\section{Interaction of Liposomes with drugs:}

Out of variety of doping agents, the direct addition of liposomes to urine samples containing anabolic steroids showed strong tendency to interact which results in the reduced concentration of compound in the sample.

Effect of Concentration of liposomes on binding efficiency:

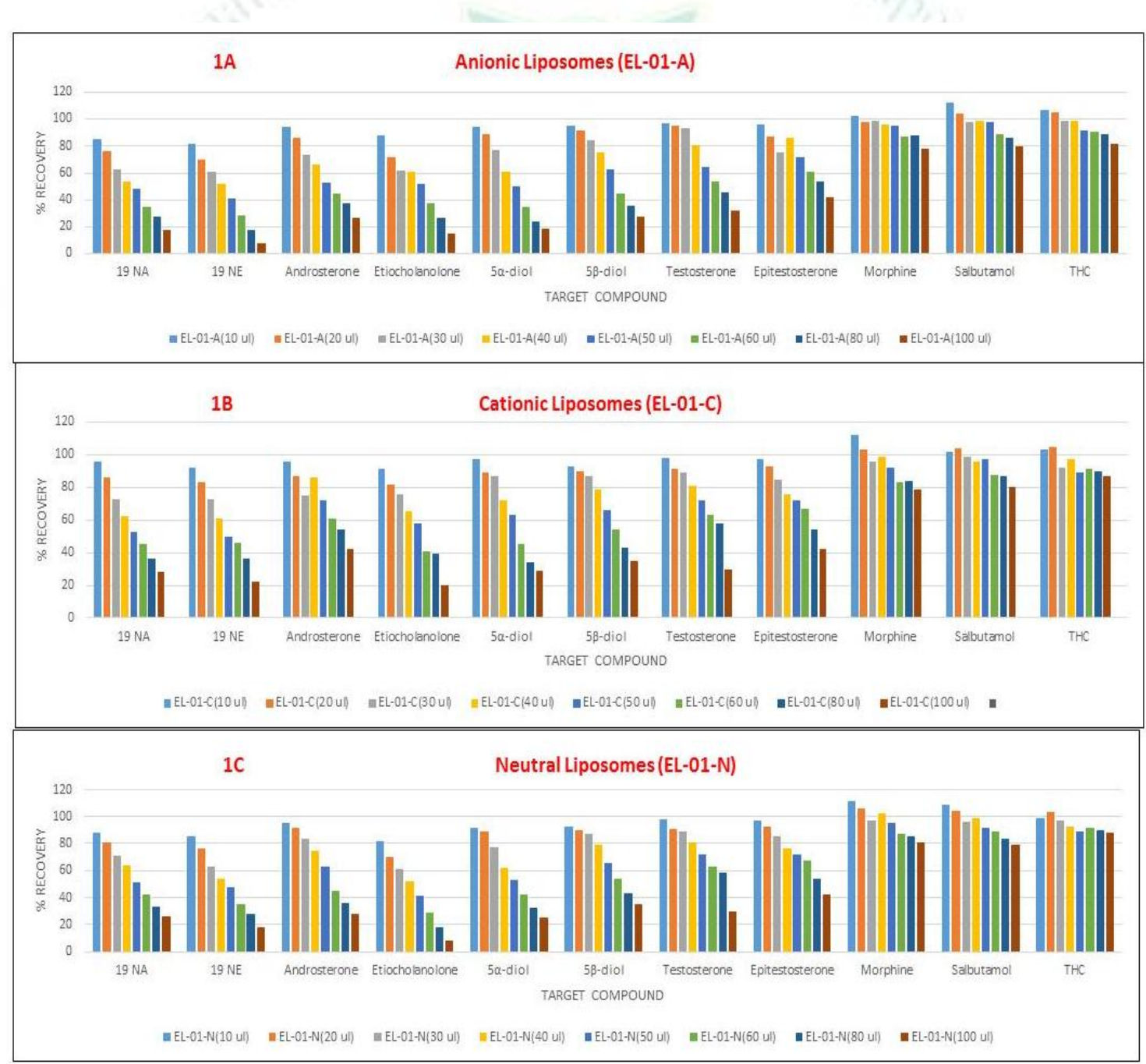

Figure 1: Effect of increasing concentration of reconstituted liposomes (10-100 $\mu \mathrm{l})$ (1A-Anionic, 1B-Cationic, 1CNeutral) on recovery of various drugs 
The recovery of various drugs in urine decreased with increasing concentration of liposomes in the urine. Out of three different formulations of liposomes, anionic showed maximum binding capacity with doping agents. However, anabolic steroids showed maximum tendency to interact with liposomes as most of the steroids are derivatives of cholesterol. The interaction of other drugs like morphine, salbutamol and CarboxyTetrahydrocannabinol (THC) with liposomes was minimal. (Fig. 1).

\section{Effect of pH on binding efficiency:}

Results of urine samples having different $\mathrm{pH}(5,7$ and 9) spiked with reconstituted liposome $(40 \mu \mathrm{l})$ and drugs revealed that basic $\mathrm{pH}$ facilitated the interaction as recovery of steroids was 5 to $10 \%$ less in basic $\mathrm{pH}$ as compared to neutral or acidic $\mathrm{pH}$. Significance of data was calculated by statistical analysis using SPSS 16.0 by applying one-way analysis of variance with posthoc Bonferroni analysis. $\mathrm{P}$ value observed was .001 which is below $\mathrm{P}<0.05$, hence considered as significant.

Effect of incubation temperature and time on binding efficiency:

No significant effect of Temperature and time on the binding efficiency of liposomes with doping agents was observed

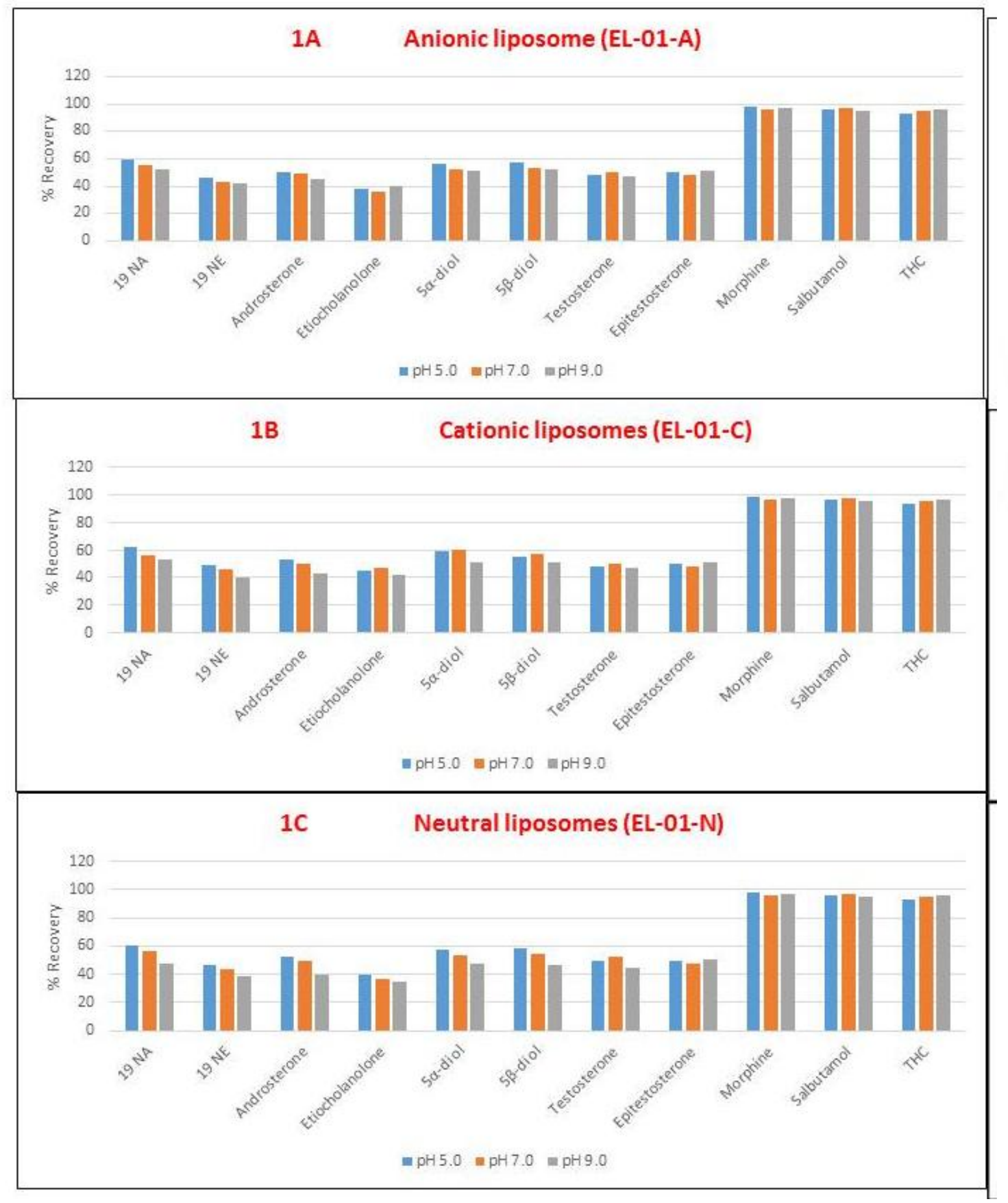

Figure 2(1): Effect of pH on interaction of liposomes (1A-Anionic, 1B-Cationic, 1C-Neutral) with various doping agents 


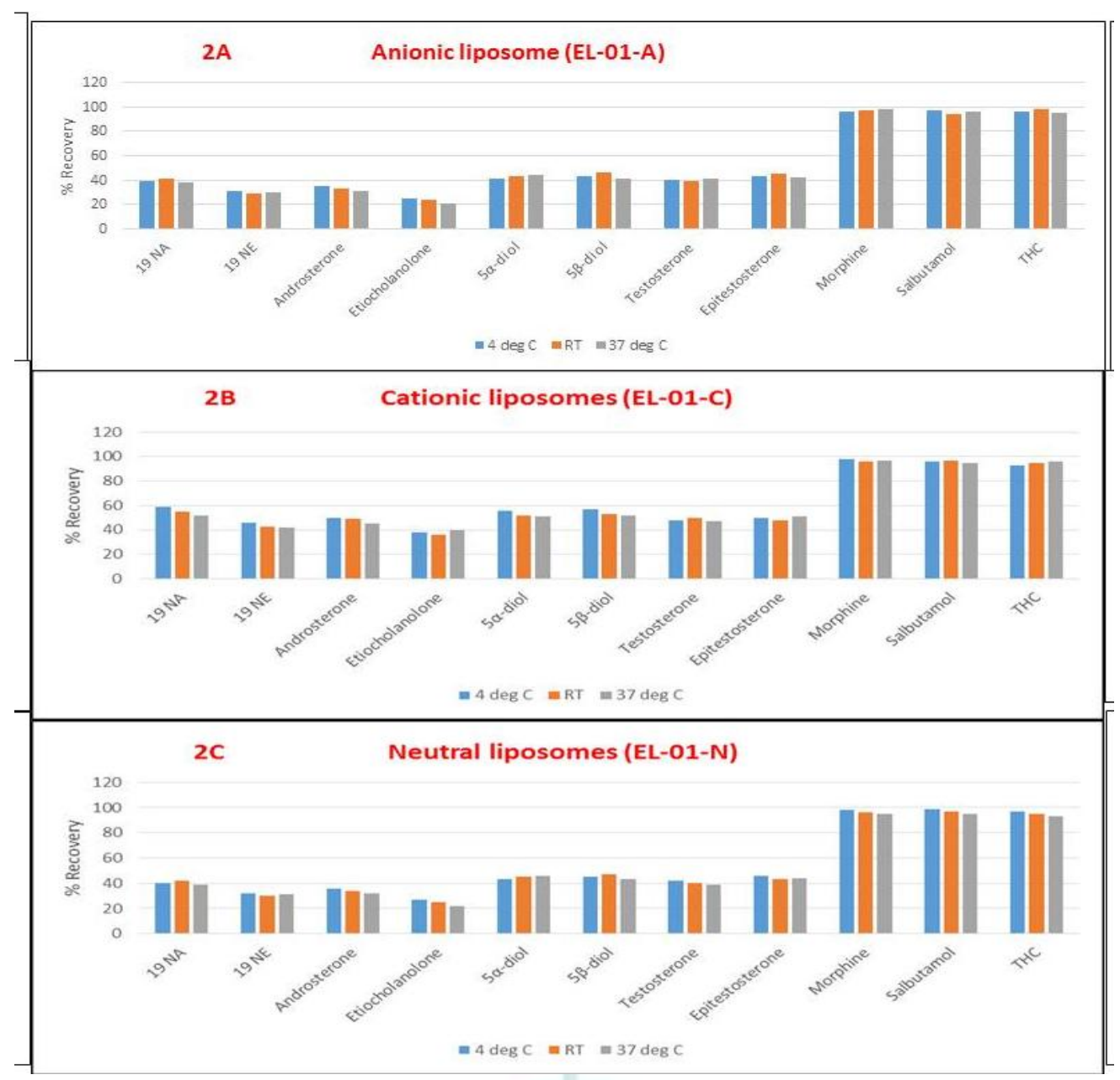

Figure 2(2): Effect of Temperature on interaction of liposomes (2A-Anionic, 2B-Cationic, 2C-Neutral) with various doping agents

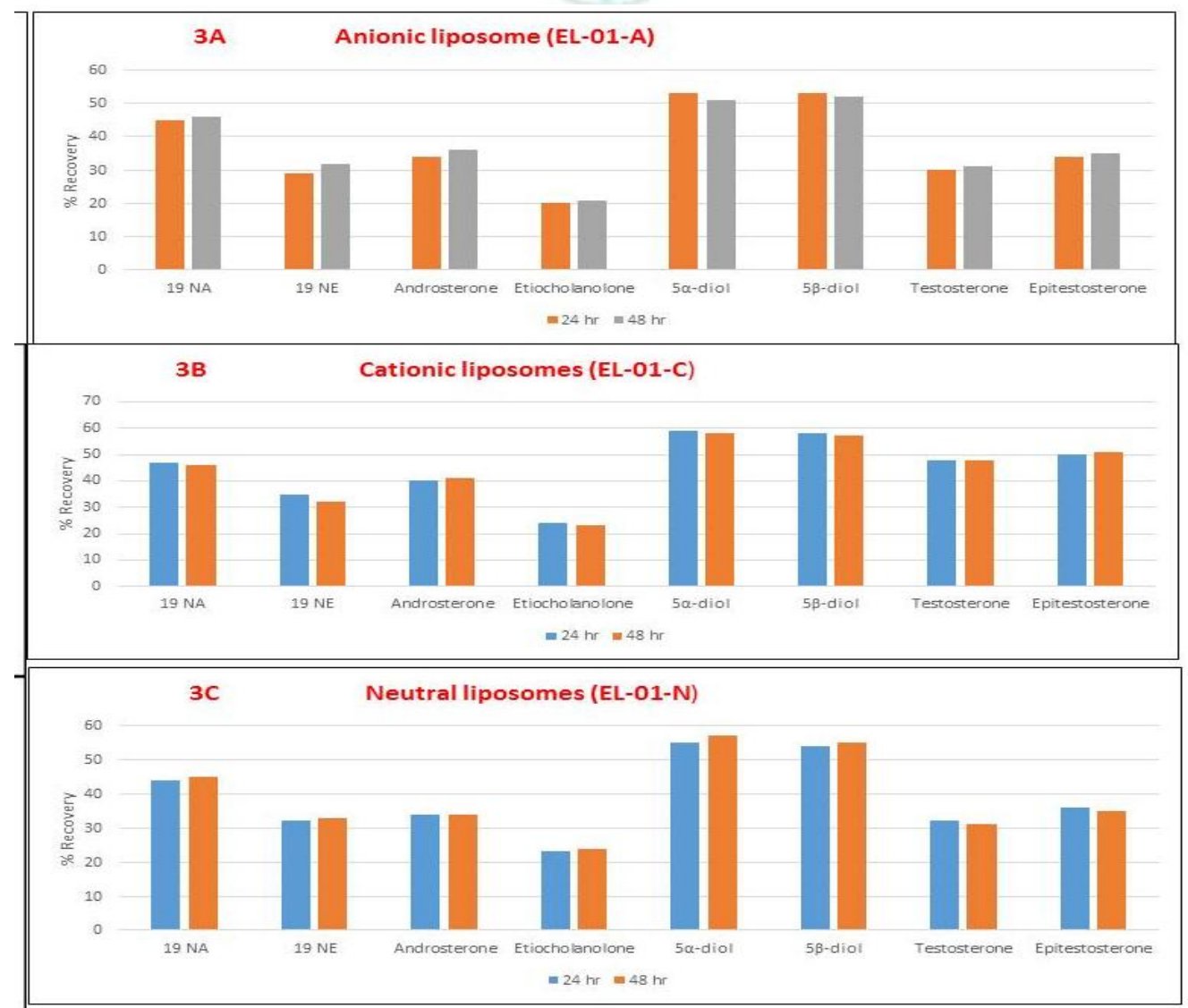

Figure 2(3): Effect of Incubation time on interaction of liposomes (3A-Anionic, 3B-Cationic, 3C-Neutral) with various doping agents 


\section{Cholesterol: Possible marker for liposome abuse}

It has been reported that cholesterol is main ingredient of liposome. It was assumed that during interaction of liposomes with various drugs, cholesterol should be released. Cholesterol is an endogenous compound and excreted in urine. Its concentration might be increased in various physiological concentration. The concentration of cholesterol was monitored in routine dope samples $(n=1000)$ and was found to be in the range of $45 \mathrm{ng} / \mathrm{ml}$ to $376 \mathrm{ng} / \mathrm{ml}$ (Fig-3). The concentrations of cholesterol were verified on each vial of liposomes used in the study by direct liposome analysis and during the experiments liposomes were spiked in urine at a concentration of $15 \mu \mathrm{g} / \mathrm{ml}$ of cholesterol. Concentration of cholesterol was quantified in blank urine $(n=5)$ and urine spiked with different formulations of liposomes. Results revealed that the concentration of cholesterol increased 4 to 5 folds when same urine samples were spiked with liposome (Fig. 3). The result of this preliminary study postulate that detection of cholesterol in routine doping sample can act as marker for abuse of liposome as masking agent.

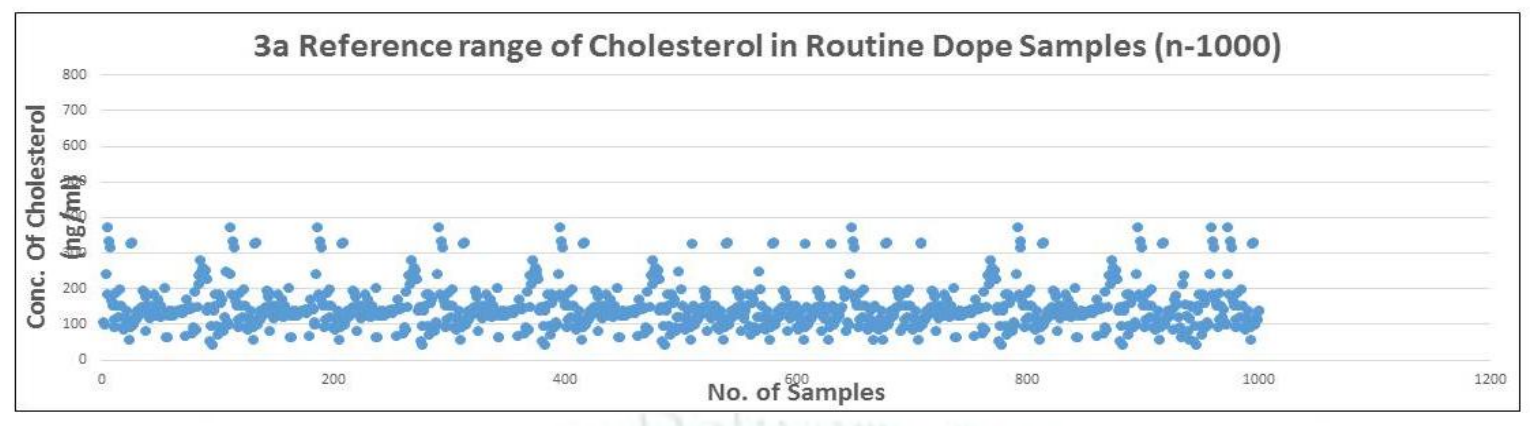

Figure 3a: Concentration of cholesterol in routine dope testing samples $(n=1000)$

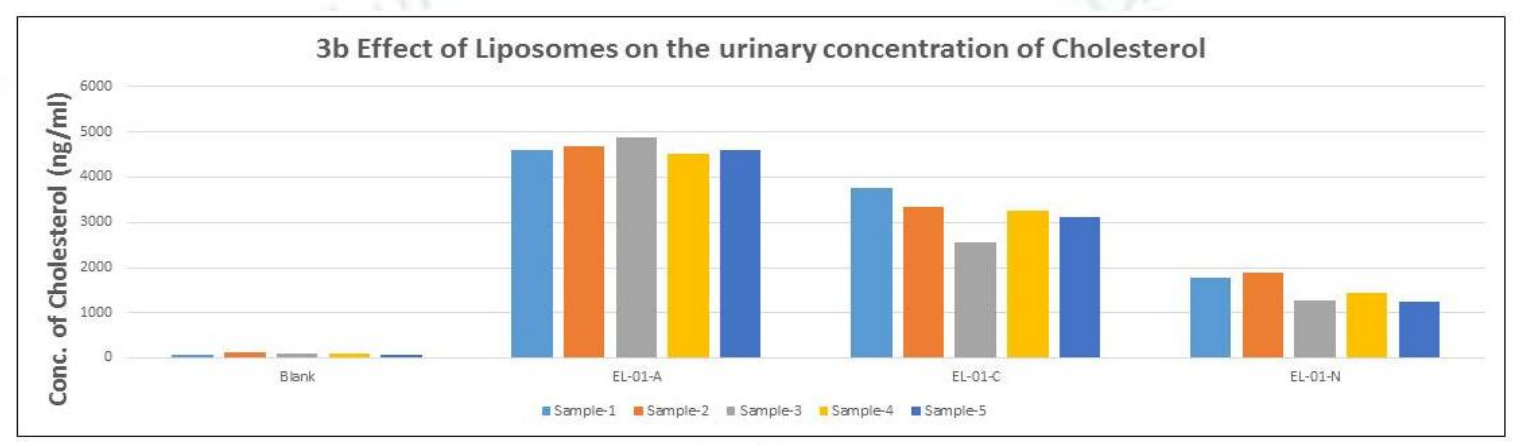

Figure 3b: Effect of liposomes on the urinary concentration of cholestrol

\section{CONCLUSION:}

This study reveals that liposome have the ability to mask different classes of drug in the urine matrix. However, androgenic anabolic steroids showed strong affinity towards liposomes when added in urine. The masking of drugs with liposome is proportional to the concentration of liposome added in the sample. Elevated $\mathrm{pH}(\mathrm{pH}$ 9) facilitated interaction of liposomes with all classes of drugs studied in the present work.

Cholesterol seems to be a potential marker of liposomes abuse with elevated level recovers in urine spiked with liposomes. However, Cholesterol is endogenously produced and may be a by-product of various physiological conditions therefore an extensive profiling of cholesterol threshold value should be established to be used as direct marker of liposome use.

\section{Acknowledgement}

The financial support of Ministry of Youth Affairs \& Sports is duly acknowledged.

\section{REFERENCES}

1. Botre F., de la Torre X., Mazzarino M., Tieri A., Bush K., Cowan D. (2009) Are liposomes masking agents? An inprogress study, In: Schanzer W, Geyer H, Gotzmann A, Mareck U, (eds.) Recent Advances in Doping Analysis (17), Koln, 2011, p 21-28.

2. Botre F., Esposito S., de la Torre X. (2011) How we risk: liposomes and steroids, In: Schanzer W, Geyer H, Gotzmann A, Mareck U, (eds.) Recent Advances in Doping Analysis (19), Koln, 2011, p 24-33.

3. Jain S., Lal R., Garg T., Jamal H., Goswami M., Nimker V., Soni A., Beotra A., Kaur T (2011) "Comparative study of endogeneous steroid profile of Indian sports person with other Commonwealth games 2010 sportsperson", In: Schanzer W, Geyer H, Gotzmann A, Mareck U, (eds.) Recent Advances in Doping Analysis (19), Koln, p 190-194. 